\title{
Histone demethylase KDM6B has an anti- tumorigenic function in neuroblastoma by promoting differentiation
}

\author{
Liqun Yang ${ }^{1}$, Yunhong Zha ${ }^{2}$, Jane Ding ${ }^{3}$, Bingwei Ye ${ }^{3}$, Mengling Liu², Chunhong Yan ${ }^{3,4}$, Zheng Dong ${ }^{5,6}$,
} Hongjuan Cui ${ }^{1}$ and Han-Fei Ding (i] $^{3,4,7}$

\begin{abstract}
Induction of differentiation is a therapeutic strategy in high-risk neuroblastoma, a childhood cancer of the sympathetic nervous system. Neuroblastoma differentiation requires transcriptional upregulation of neuronal genes. How this process is regulated at epigenetic levels is not well understood. Here we report that the histone H3 lysine 27 demethylase KDM6B is an epigenetic activator of neuroblastoma cell differentiation. KDM6B mRNA expression is downregulated in poorly differentiated high-risk neuroblastomas and upregulated in differentiated tumors, and high KDM6B expression is prognostic for better survival in neuroblastoma patients. In neuroblastoma cell lines, KDM6B depletion promotes cell proliferation, whereas KDM6B overexpression induces neuronal differentiation and inhibits cell proliferation and tumorgenicity. Mechanistically, KDM6B epigenetically activates the transcription of neuronal genes by removing the repressive chromatin marker histone $\mathrm{H} 3$ lysine 27 trimethylation. In addition, we show that KDM6B functions downstream of the retinoic acid-HOXC9 axis in inducing neuroblastoma cell differentiation: KDM6B expression is upregulated by retinoic acid via HOXC9, and KDM6B is required for HOXC9-induced neuroblastoma cell differentiation. Finally, we present evidence that KDM6B interacts with HOXC9 to target neuronal genes for epigenetic activation. These findings identify a KDM6B-dependent epigenetic mechanism in the control of neuroblastoma cell differentiation, providing a rationale for reducing histone H3 lysine 27 trimethylation as a strategy for enhancing differentiation-based therapy in high-risk neuroblastoma.
\end{abstract}

\section{Introduction}

Neuroblastoma is a common pediatric cancer of the sympathetic nervous system derived from the neural crest cell $^{1-4}$. Approximately half of all neuroblastoma cases are classified as high risk ${ }^{5}$, which have an overall survival rate $<50 \%$, even after intensive, multimodal therapy ${ }^{6,7}$. Highrisk neuroblastomas are predominantly Schwannian

\footnotetext{
Correspondence: Hongjuan Cui (hcui@swu.edu.cn) or Han-Fei Ding (hding@augusta.edu)

'State Key Laboratory of Silkworm Genome Biology, Southwest University, Chongqing 400716, China

${ }^{2}$ Institute of Neural Regeneration and Repair and Department of Neurology, The First Hospital of Yichang, Three Gorges University College of Medicine, Yichang 443000, China

Full list of author information is available at the end of the article.

These authors contributed equally: Liqun Yang, Yunhong Zha
}

stroma-poor, undifferentiated or poorly differentiated tumors ${ }^{5,8}$, and induction of differentiation by agents, such as all trans-retinoic acid (RA $)^{9,10}$, has been exploited as a treatment strategy in high-risk neuroblastoma ${ }^{11-14}$. A better understanding of the molecular basis of neuroblastoma differentiation may suggest new therapeutic targets or strategies for enhancing the efficacy of differentiation-based therapy for high-risk neuroblastoma patients.

Studies of normal stem cell differentiation have demonstrated a pivotal role of epigenetic regulation via histone lysine methylation in the differentiation process $^{15,16}$. For example, trimethylation of histone $\mathrm{H} 3$ at lysine 27 (H3K27me3) is required for the transcriptional repression of development genes and the maintenance of

\section{(c) The Author(s) 2019}

(c) (i) Open Access This article is licensed under a Creative Commons Attribution 4.0 International License, which permits use, sharing, adaptation, distribution and reproduction c. in any medium or format, as long as you give appropriate credit to the original author(s) and the source, provide a link to the Creative Commons license, and indicate if changes were made. The images or other third party material in this article are included in the article's Creative Commons license, unless indicated otherwise in a credit line to the material. If material is not included in the article's Creative Commons license and your intended use is not permitted by statutory regulation or exceeds the permitted use, you will need to obtain permission directly from the copyright holder. To view a copy of this license, visit http://creativecommons.org/licenses/by/4.0/. 


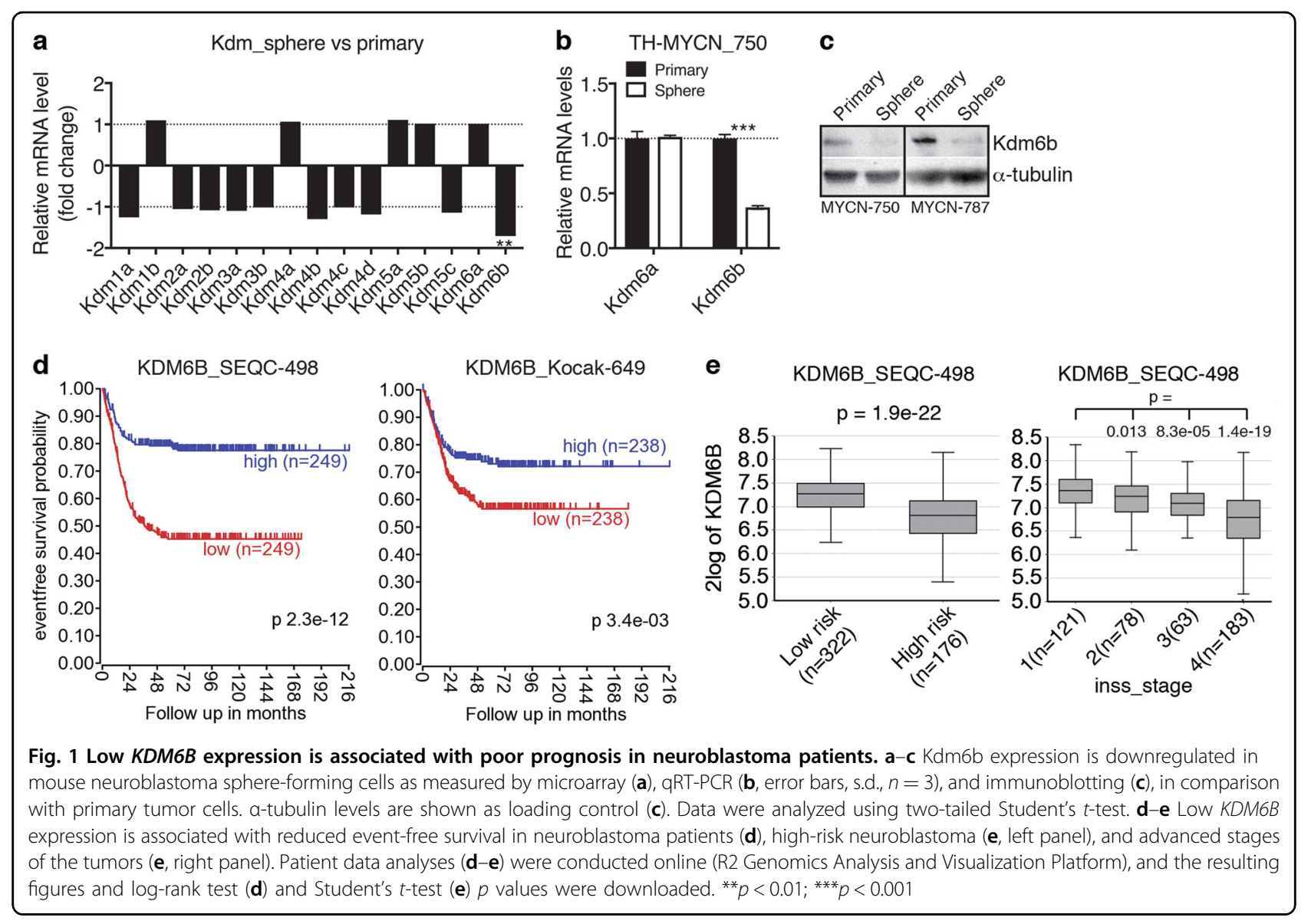

pluripotency in embryonic stem cells (ESCs) ${ }^{17,18}$. Interestingly, recent studies have provided evidence for an important role of the H3K27-specific methyltransferase $\mathrm{EZH} 2$ in neuroblastoma pathogenesis. It has been reported that high EZH2 expression is prognostic for poor clinical outcome in neuroblastoma patients and that EZH2-catalyzed H3K27me3 is responsible for transcriptional repression of several neuroblastoma tumorsuppressor genes, including CASZ1 GLU, RUNX3, and $N G F R^{19}$. In addition, Ezh2 activity has been shown to be critical for the growth of mouse neuroblastoma spheres in culture and for tumor development in the TH-MYCN mouse mode ${ }^{20}$. In line with these findings, a more recent study has revealed a crucial role of EZH2 in blocking neuroblastoma cell differentiation ${ }^{21}$.

Since histone lysine methylation levels are determined by the balance between the activities of histone lysine methyltransferases and demethylases ${ }^{22,23}$, we reasoned that histone lysine demethylases (KDMs) that antagonize the activity of EZH2 by removing H3K27me3 might have an onco-suppressor function in neuroblastoma. The KDM6B family of demethylases KDM6A and KDM6B, also commonly known as UTX and JMJD3, respectively, are responsible for the removal of $\mathrm{H} 3 \mathrm{~K} 27 \mathrm{me} 3^{23,24}$. Our investigation provides evidence in support of this model, revealing an anti-tumorigenic activity of KDM6B in neuroblastoma cells by inducing neuronal differentiation.

\section{Results \\ $K D M 6 B$ expression is downregulated in neuroblastoma stem-like cells and in high-risk neuroblastoma}

We recently reported the isolation and propagation of a population of neuroblastoma sphere-forming cells with cancer stem cell activities, including self-renewal capacity and increased tumorigenic potential, from tumors of the TH-MYCN mouse ${ }^{25}$, an animal model of high-risk neuroblastoma with $M Y C N$ amplification ${ }^{26-29}$. To assess the functions of KDMs in determining neuroblastoma differentiation states, we examined the microarray gene expression profiling data from three independent lines of sphere-forming cells (stem cell state) in comparison with their parental primary tumors (differentiated state) ${ }^{25}$. Strikingly, $K d m 6 b$ expression was selectively downregulated in sphere-forming cells compared to primary tumor cells, as no significant changes were observed in the expression levels of other demethylase genes examined (Fig. 1a). We verified the observation by quantitative reverse transcriptase-PCR (qRT-PCR) and immunoblot 
analyses, which showed downregulation of $K d m 6 b$, but not $K d m 6 a$, mRNA expression (Fig. 1b) and of Kdm6b protein expression (Fig. 1c) in sphere-forming cells relative to their parental primary tumors. Thus, mouse neuroblastoma stem-like cells express significantly lower levels of Kdm6b compared to their more differentiated primary tumor cells.

To assess the clinical relevance of this observation, we examined the correlation between $K D M 6 B$ mRNA expression levels and clinical outcomes using the gene expression profiling data from two independent cohorts of neuroblastoma patients $(n=1147)^{30,31}$. We found that lower $K D M 6 B$ expression is significantly associated with reduced event-free survival of neuroblastoma patients (Fig. 1d), with high-risk neuroblastoma tumors (Fig. 1e, left panel), and with advanced tumor stages (Fig. 1e, right panel). By contrast, KDM6A mRNA expression levels showed no correlation with the survival of neuroblastoma patients (Supplementary Fig. 1). Taken together, these results suggest that KDM6B might function as an epigenetic onco-suppressor in the pathogenesis of high-risk neuroblastoma.

\section{KDM6B inhibits neuroblastoma cell proliferation and tumorigenicity}

Given that high $K D M 6 B$ expression is indicative of better prognosis for neuroblastoma patients (Fig. 1d), we investigated the effect of high KDM6B expression on neuroblastoma cell proliferation and tumorigenicity. Ectopic expression of human KDM6B (Fig. 2a) markedly inhibited the proliferation of both $M Y C N$-amplified (BE (2)-C and SMS-KCNR) and non-MYCN-amplified (SHSY5Y and SK-N-AS) human neuroblastoma cell lines (Fig. $2 \mathrm{~b})$. Overexpression of the demethylase-defective mutant KDM6B-H1390A did not significantly inhibit the growth of SK-N-AS and BE(2)-C cells (Fig. 2a, b, KDM6Bmut), indicating that the demethylase activity of KDM6B is required for its growth-inhibitory effect. Similarly, ectopic expression of human KDM6B in mouse neuroblastoma sphere-forming cells resulted in a marked inhibition of cell growth (Fig. 2c). In line with these results, BE(2)-C and SK-N-AS cells with KDM6B overexpression generated much smaller tumors in immunodeficient mice relative to their vector control counterparts (Fig. 2d), indicating that high KDM6B expression reduced tumorigenicity of neuroblastoma cells.

To assess the effect of endogenous KDM6B expression on neuroblastoma cell proliferation, we used two distinct short hairpin RNA (shRNA) lentiviral constructs to reduce $\mathrm{KDM} 6 \mathrm{~B}$ expression (Fig. 2e, f), which had no significant effect on KDM6A expression (Fig. 2e). Knockdown of KDM6B expression significantly enhanced the proliferation of $\mathrm{BE}(2)-\mathrm{C}$ and SMS-KCNR cells (Fig. $2 \mathrm{~g})$. Collectively, these results reveal a growth-inhibitory and anti-tumorigenic function of KDM6B in neuroblastoma cells.

\section{KDM6B promotes neuronal differentiation of neuroblastoma cells}

To gain a molecular understanding of the antitumorigenic activity of KDM6B in neuroblastoma cells, we performed gene ontology (GO) analysis of the genes that are co-expressed with $K D M 6 B$ in a cohort of 498 neuroblastoma patients (the SEQC dataset) ${ }^{30}$ (Supplementary Table S1). Our analysis revealed a positive correlation in mRNA expression between $K D M 6 B$ and genes involved in ARF protein signaling, small GTPase signaling, actin cytoskeleton organization, axon genesis, and neuronal differentiation (Supplementary Fig. 2 and Table S2). Of note, the ARF and RAS families of small GTPases have a key role in regulation of cytoskeleton reorganization that is essential for neurogenesis and neurite outgrowth, branching, and retraction ${ }^{32-35}$. Thus, high KDM6B expression is associated with neuroblastoma differentiation. In agreement with the genetic evidence, overexpression of KDM6B in several neuroblastoma cell lines induced neuronal differentiation characterized by extensive neurite outgrowth (Fig. 3a and Supplementary Fig. 3a). We further confirm that these cells underwent neuronal differentiation at the molecular level by qRTPCR, which showed that overexpression of KDM6B, but not its demethylase-defective mutant, resulted in a significant increase in mRNA levels of neuronal and differentiation marker genes, including neurofilament medium (NEFM), glial cell line-derived neurotrophic factor (GDNF) family receptor alpha 3 (GFRA3), and RET (Fig. $3 b)$. In addition, immunoblot analysis revealed that overexpression of $\mathrm{KDM} 6 \mathrm{~B}$, but not its mutant, increased NEFM protein levels in neuroblastoma cells (Fig. 3c). It is known that expression of these genes is increased in neuroblastoma cells undergoing neuronal differentiation induced by RA and HOX proteins ${ }^{36-40}$. NEFM is upregulated during neuronal development and is involved in axon outgrowth and guidance ${ }^{41}$, and GFRA3 forms a receptor complex with RET, which has a critical role in promoting the survival, differentiation, axonal outgrowth, and target innervation of sympathetic neurons ${ }^{42}$.

We next investigated whether KDM6B directly targets neuronal genes, using NEFM as a model system. Chromatin immunoprecipitation and quantitative PCR (ChIPqPCR) revealed that $\mathrm{KDM} 6 \mathrm{~B}$ overexpression in $\mathrm{BE}(2)-\mathrm{C}$ cells led to a marked increase in KDM6B levels at the promoter of $N E F M$ (Fig. 3d), which was accompanied by a significant reduction in $\mathrm{H} 3 \mathrm{~K} 27 \mathrm{me} 3$ levels in the promoter region (Fig. 3e). Of note, KDM6B overexpression or downregulation had no significant effect on global H3K27me3 levels (Supplementary Fig. 3b). These observations are consistent with the notion that KDM6B has a 




key role in the removal of H3K27me3 from lineage genes during differentiation, but has no significant impact on genome-wide H3K27me3 deposition ${ }^{43}$. Collectively, these findings suggest that high levels of KDM6B initiate a neuronal differentiation program in neuroblastoma cells by conferring an epigenetically active chromatin state to neuronal genes for transcriptional activation.

\section{KDM6B is induced by RA and is a direct transcriptional target of HOXC9}

The above observation that KDM6B overexpression was sufficient to induce neuroblastoma cell differentiation prompted us to investigate whether KDM6B has a role in the action of RA, which is commonly used for induction of neuroblastoma cell differentiation ${ }^{9,10}$ and has been used in clinic for treatment of high-risk neuroblastomas ${ }^{11-14}$. We have shown previously that HOXC9 is a major mediator of RA action in inducing neuroblastoma cell differentiation ${ }^{38-40}$. As expected, RA treatment resulted in upregulation of HOXC9 and neuronal genes, including GFRA3, NEFM, and RET (Fig. 4a). We found that RA treatment also increased KDM6B mRNA and protein expression in a time-dependent manner (Fig. 4a, b). Importantly, RA induction of $K D M 6 B$ mRNA was 


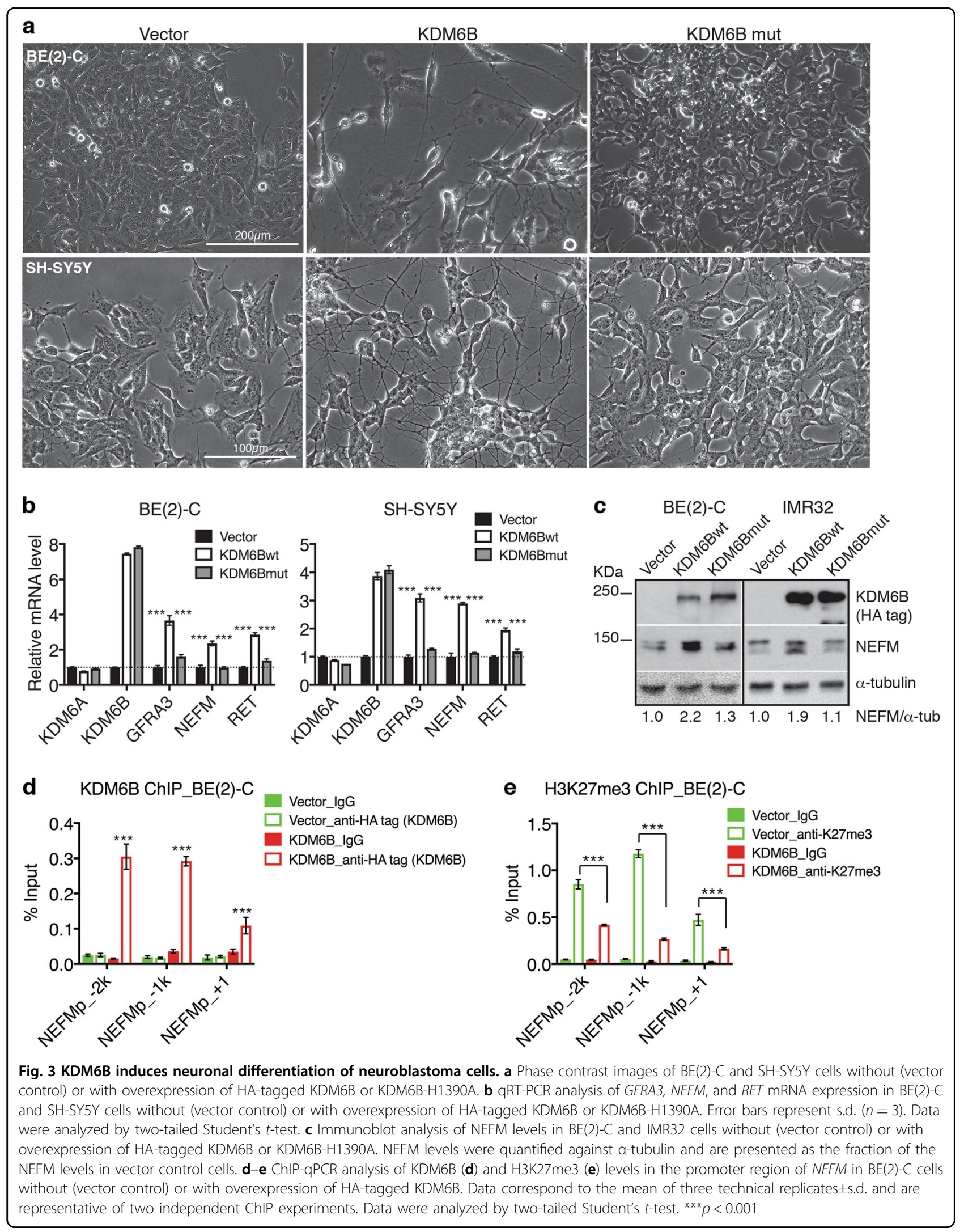




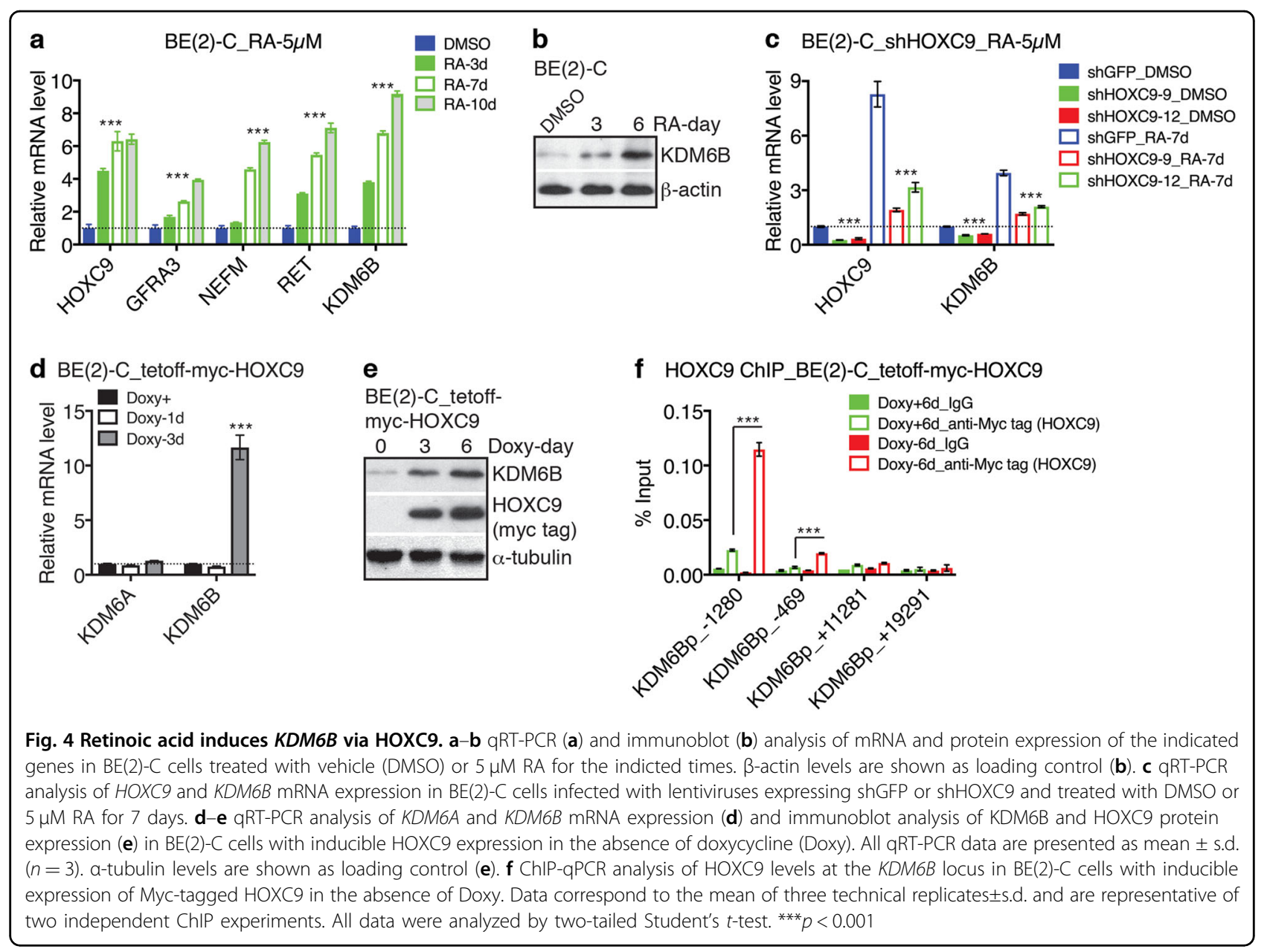

dependent on HOXC9 since knockdown of HOXC9 expression by shRNA significantly reduced the levels of $K D M 6 B$ induction (Fig. 4c).

We noticed that HOXC9 knockdown alone led to a significant decrease in $K D M 6 B$ mRNA levels in the absence of RA treatment (Fig. 4c), indicating an essential role of HOXC9 in maintaining the steady-state level of KDM6B mRNA expression in neuroblastoma cells. Moreover, HOXC9 overexpression was sufficient to induce KDM6B mRNA and protein expression (Fig. 4d, e), but had no effect on KDM6A mRNA expression (Fig. 4d). In agreement with these experimental data, we found that higher HOXC9 mRNA expression is significantly associated with higher $K D M 6 B$ mRNA expression in primary neuroblastoma tumors (Supplementary Figure 4). By contrast, there is no significant correlation in $\mathrm{HOXC9}$ and $K D M 6 A$ expression in these tumors (Supplementary Figure 4).

HOX proteins bind DNA sequences with a consensus TAATT/AA-motif ${ }^{44,45}$. In BE(2)-C cells with HOXC9 overexpression, ChIP-qPCR analysis detected significant levels of HOXC9 associated with the promoter of
$K D M 6 B$, particularly around the potential HOX-binding sequence TAATTG starting at 1232 bases upstream of the transcription start site $(+1)$ (Fig. $4 \mathrm{f})$. We found no significant levels of HOXC9 enrichment in other regions of the $K D M 6 B$ locus examined, including exon 5 that encodes the first 46 amino acids (Fig. 4f, KDM6Bp +11281 ) and the $3^{\prime}$ untranslated region (Fig. 4f, KDM6Bp_+19291). Thus, HOXC9 binding to the promoter region of $K D M 6 B$ is highly specific. Taken together, these data provide evidence for $K D M 6 B$ as a direct transcriptional target gene of HOXC9 in RA-induced neuronal differentiation of neuroblastoma cells.

\section{KDM6B is required for HOXC9-induced neuronal differentiation of neuroblastoma cells}

HOXC9 is induced by RA and is a major downstream mediator of RA action, and HOXC9 overexpression is sufficient to induce neuronal differentiation of neuroblastoma cells ${ }^{38,39}$. Since HOXC9 activates $K D M 6 B$ transcription, we asked whether KDM6B upregulation is required for HOXC9-induced differentiation. We used shRNA lentiviral constructs to reduce KDM6B mRNA 

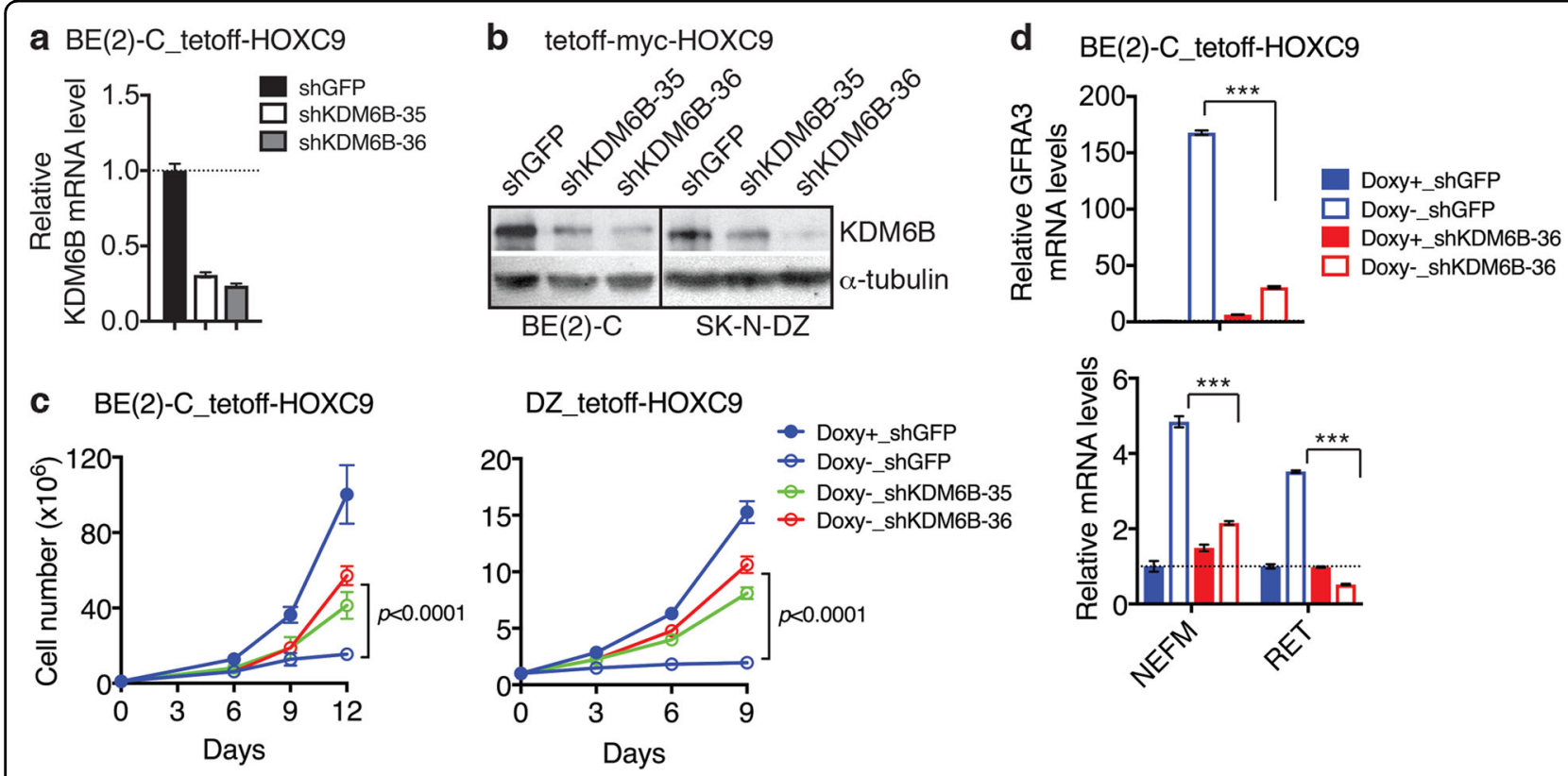

Fig. 5 KDM6B is required for HOXC9-induced neuronal differentiation. $\mathbf{a}-\mathbf{b}$ qRT-PCR (a) and immunoblot (b) analyses of KDM6B mRNA and protein expression in BE(2)-C_ and SK-N-DZ_tetoff-myc-HOXC9 cells infected with lentiviruses expressing shGFP or shKDM6B. Error bars represent s.d. $(n=3)$. a-tubulin levels are shown as loading control $(\mathbf{b})$. c Growth curves of BE(2)-C_ and SK-N-DZ_tetoff-myc-HOXC9 cells expressing either shGFP or shKDM6B-36 that were cultured in the presence or absence of Doxy for the indicted times. Data correspond to the mean of four technical replicates \pm s.d. and are representative of at least two independent experiments. Data were analyzed by two-way ANOVA with $p$ values indicated. d qRT-PCR analysis of GFRA3, NEFM, and RET mRNA expression in BE(2)-C_tetoff-myc-HOXC9 cells expressing either shGFP or shKDM6B-36 that were cultured in the presence or absence of doxycycline (Doxy) for 9 days. Data were analyzed by two-tailed Student's $t$-test. ${ }^{* * *} p<0.001$

and protein expression in neuroblastoma cell lines with inducible HOXC9 expression (Fig. 5a, b). As expected ${ }^{38,39}$, $\mathrm{BE}(2)-\mathrm{C}$ ttetoff-myc-HOXC9 cells cultured in the absence of doxycycline underwent neuronal differentiation, showing growth arrest and extensive neurite outgrowth (Fig. 5c and Supplementary Fig. 5). Knockdown of KDM6B expression blocked HOXC9-induced differentiation, as evidenced by the lack of growth arrest and neurite outgrowth of shKDM6B-expressing cells following HOXC9 induction (Fig. 5c and Supplementary Fig. 5). We obtained similar results with SK-N-DZ_tetoff-mycHOXC9 cells (Fig. 5c). At the molecular level, KDM6B knockdown largely abolished the ability of HOXC9 to upregulate neuronal genes, including GFRA3, NEFM, and RET (Fig. 5d). These findings indicate that KDM6B is a key mediator of HOXC9 action in induction of neuroblastoma cell differentiation.

\section{KDM6B interacts with and requires HOXC9 to target neuronal genes for epigenetic activation}

To gain further understanding of the molecular basis of KDM6B action in promoting differentiation, we investigated how KDM6B is targeted to neuronal genes during HOXC9-induced differentiation, again using the NEFM promoter as a model. We have shown previously that HOXC9 binds to the consensus TAATTA sequence located $\sim 1.9$-kb upstream of the NEFM gene transcription start site $^{38}$. As expected, HOXC9 overexpression led to a marked increase in HOXC9 levels at the NEFM promoter (Fig. 6a, blue columns). Importantly, the levels of endogenous KDM6B at the NEFM promoter were also significantly increased (Fig. 6a, red columns), accompanied by a marked reduction in H3K27me3 levels (Fig. 6a, green columns). Thus, HOXC9 overexpression increases KDM6B binding to the NEFM promoter.

In parallel studies, we used shRNA lentiviral constructs to downregulate $\mathrm{HOXC9}$ in $\mathrm{BE}(2)-\mathrm{C}$ cells overexpressing KDM6B. As shown above (Fig. 3d), KDM6B overexpression resulted in a significant increase in KDM6B levels at the NEFM promoter (Fig. 6b). This increase was significantly abolished by knockdown of HOXC9 expression (Fig. 6b). Moreover, we found that endogenous KDM6B could be co-immunoprecipitated with HOXC9 under the condition of HOXC9 overexpression (Fig. 6c). Together, these results suggest that HOXC9 interacts with and recruits KDM6B to neuronal gene promoters for epigenetic activation of gene expression (Fig. 7).

\section{Discussion}

In this report, we present evidence for KDM6B being an epigenetic activator of neuroblastoma cell differentiation. We show that $K D M 6 B$ is downregulated in 
a ChIP_BE(2)-C_tetoff-myc-HOXC9

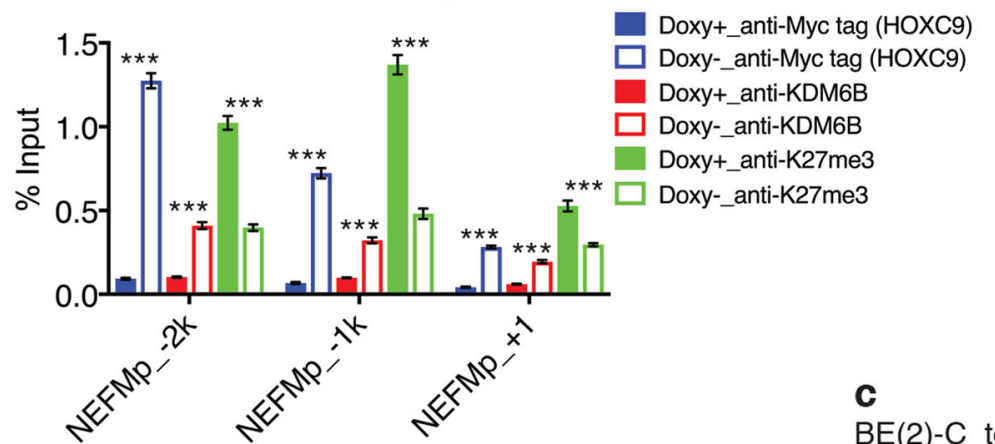

BE(2)-C_tetoff-Myc-HOXC9

Doxy-6d

b KDM6B ChIP_BE(2)-C-KDM6B
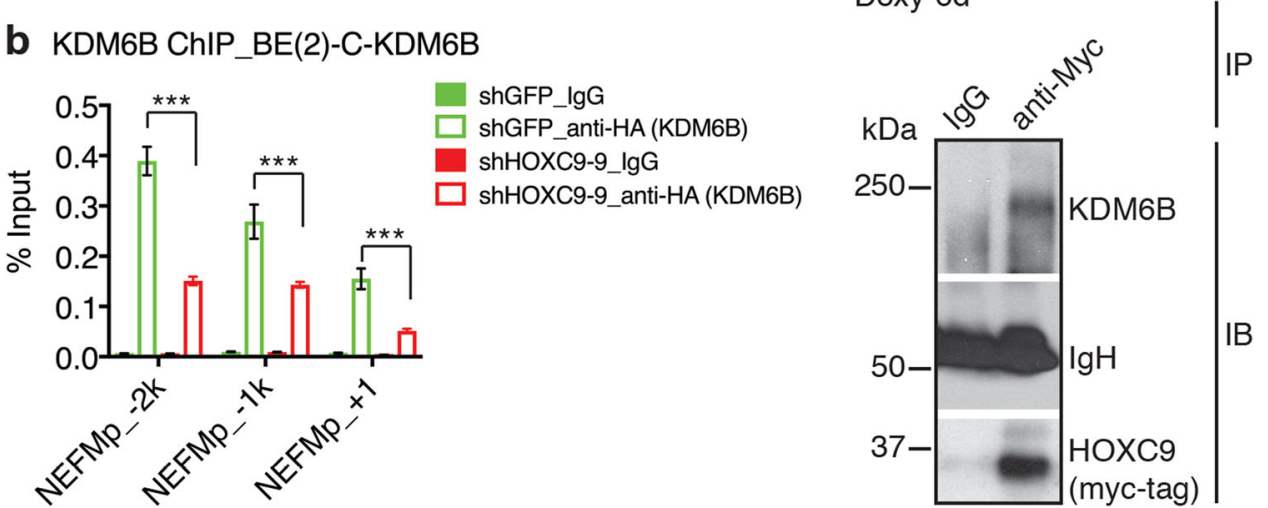

Fig. 6 KDM6B interacts with HOXC9 for targeting neuronal genes for epigenetic activation. a ChIP-qPCR analysis of HOXC9, KDM6B, and H3K27me3 levels in the promoter region of NEFM in BE(2)-C_tetoff-myc-HOXC9 cells that were cultured in the presence of absence of Doxy for 6 days. $\mathbf{b}$ ChIP-qPCR analysis of KDM6B levels in the promoter region of NEFM in BE(2)-C cells with overexpression of HA-tagged KDM6B without (shGFP) or with HOXC9 knockdown by shHOXC9-9. All ChIP-qPCR data correspond to the mean of three technical replicates \pm s.d. and are representative of two independent ChIP experiments. Data were analyzed by two-tailed Student's $t$-test. *** $p<0.001$. c Co-IP showing HOXC9 interaction with endogenous KDM6B in BE(2)-C_tetoff-myc-HOXC9 cells following HOXC9 induction in the absence of Doxy for 6 days. IgH immunoglobulin heavy chain

neuroblastoma stem-like cells and in poorly differentiated, high-risk neuroblastoma tumors. Moreover, higher $K D M 6 B$ expression is correlated with higher expression of genes that promote neuronal differentiation and is prognostic for better survival in neuroblastoma patients. Finally, in a panel of neuroblastoma cell lines, we show that KDM6B depletion enhanced cell proliferation, whereas KDM6B overexpression induced neuronal differentiation and inhibited the proliferation and tumorigenicity of neuroblastoma cells. Collectively, these findings suggest an onco-suppressor function of KDM6B in neuroblastoma pathogenesis by promoting differentiation. Our findings, together with those from recent studies on EZH $2^{19-21}$, indicate that modulation of the H3K27 methylation status is a key epigenetic event in neuroblastoma development.

Induction of differentiation by RA is a therapeutic strategy against high-risk neuroblastoma ${ }^{11,12}$, which, in combination with bone marrow transplant, can significantly improve the event-free survival of high-risk neuroblastoma patients ${ }^{13,14}$. However, resistance to RA presents a major barrier to successful RA-based therapy ${ }^{11,12}$. Identification of downstream mediators of RA action may suggest new strategies to bypass resistance to RA or to enhance its therapeutic effect. We recently identified an RA-HOXD8-HOXC9 axis in driving neuroblastoma cell differentiation: ${ }^{38-40}$ RA induces HOXD8, which, in turn, transcriptionally activates HOXC9 expression. Knockdown of either HOXD8 or HOXC9 expression confers resistance to RA-induced differentiation and forced expression of HOXD8 or HOXC9 can recapitulate the differentiation phenotype induced by RA. Data from the current study reveal an additional step in this molecular pathway, identifying KDM6B as a component of the axis downstream of HOXC9 (Fig. 7). RA induces $\mathrm{KDM} 6 \mathrm{~B}$ in a HOXC9-dependent manner, and HOXC9 binds to the $K D M 6 B$ promoter to transcriptionally activate $K D M 6 B$ expression. In turn, KDM6B epigenetically activates a neuronal differentiation program by removing the repressive marker $\mathrm{H} 3 \mathrm{~K} 27 \mathrm{me} 3$ from neuronal genes. Moreover, our data suggest that HOXC9 and KDM6B cooperate in driving this differentiation 


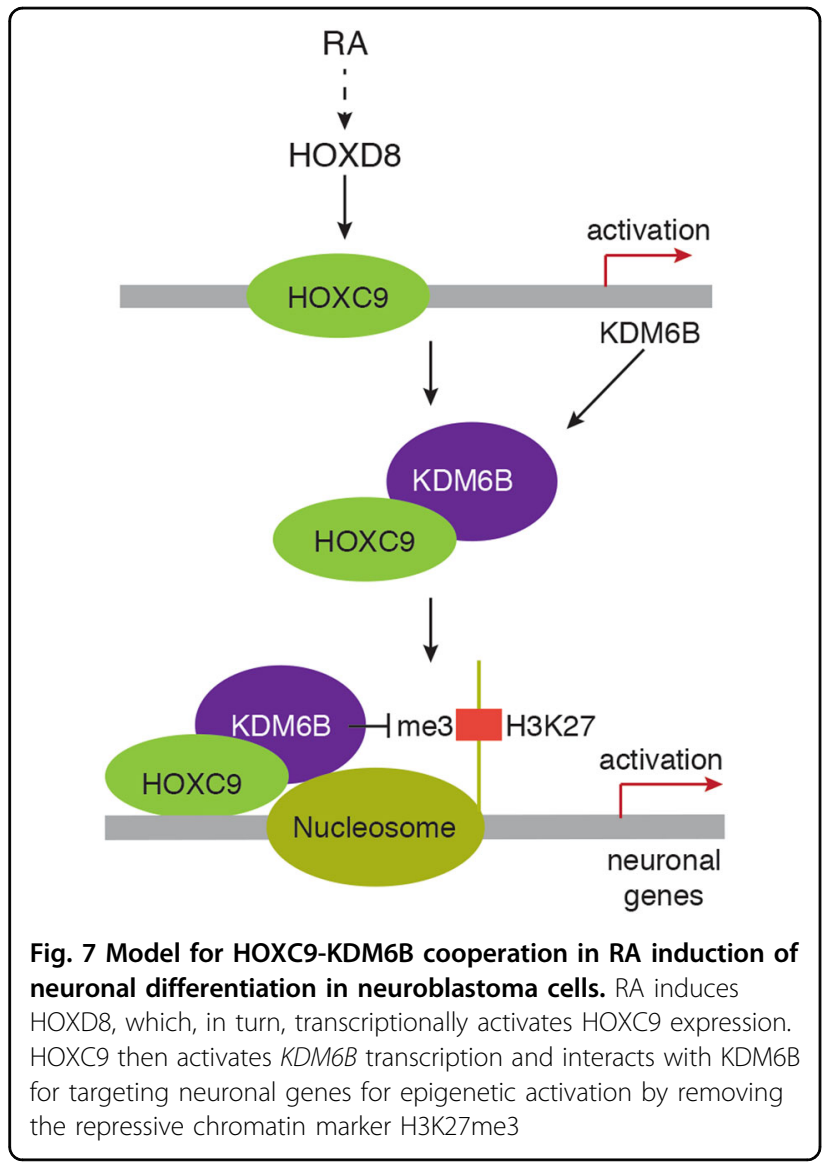

program: HOXC9 upregulates and requires KDM6B to induce neuronal genes, and KDM6B requires $\mathrm{HOXC9}$ for targeting neuronal genes for epigenetic activation.

The KDM6 family consists of two members, KDM6A and KDM6B. Although both can remove H3K27me3, our data suggest that KDM6A is unlikely to have a significant role in regulation of neuroblastoma cell differentiation. $K D M 6 A$ expression was not correlated with the differentiation state of neuroblastoma tumors and was not upregulated during neuronal differentiation of neuroblastoma cells induced by RA or HOXC9. Also, KDM6B knockdown did not induce KDM6A in neuroblastoma cells. Our observations are consistent with experimental evidence suggesting that KDM6A and KDM6B may function in different developmental lineages. For example, KDM6A is essential for cardiac differentiation of ESCs and for heart development in vivo, but is dispensable for neuronal differentiation of $\mathrm{ESCs}^{46}$. By contrast, KDM6B is required for neuronal differentiation of ESCs and neurogenesis in the brain $^{47,48}$. This lineage-dependent activity of KDM6B might also help explain why KDM6B is oncogenic in some cancers, such as T-cell acute lymphocytic leukemia ${ }^{49}$, but anti-oncogenic in neuroblastoma. This notion suggests that targeting KDM6B might offer a strategy to selectively activate differentiation of neuroblastoma cells.

In summary, our study provides clinical and experimental evidence for KDM6B as an epigenetic oncosuppressor in high-risk neuroblastoma by activating a neuronal differentiation program. The findings that either inhibition of EZH $2^{21}$ or activation of KDM6B, as reported here, was sufficient to induce neuroblastoma differentiation suggest that reducing H3K27me3 levels could be a differentiation-based therapeutic strategy in high-risk neuroblastoma, particularly in combination with RA or other differentiation-inducing agents.

\section{Materials and methods \\ Cell lines and culture}

Neuroblastoma cell lines BE(2)-C (CRL-2268), IMR32 (CCL-127), SH-SY5Y (CRL-2266), SK-N-AS (CRL-2137), and SK-N-DZ (CRL-2149) were obtained from ATCC (Manassas, VA), LA1-55n from Sigma-Aldrich (06041203, St. Louis, MO), and SMS-KCNR from the Childhood Cancer Repository at Texas Tech University Health Sciences Center. IMR32, SH-SY5Y, SK-N-AS, and SK-N-DZ were cultured in DMEM (HyClone SH30022); BE(2)-C in DME/F-12 1:1 (HyClone SH30023); and LA1-55n and SMS-KCNR in RPMI 1640 (HyClone SH30027) from Thermo Fisher Scientific (Waltham, MA). All media were supplemented with 10\% FBS (S11050, Atlanta Biologicals, Flowery Branch, GA). Mouse neuroblastoma sphereforming cell lines were established as described previously ${ }^{25}$ and were cultured in complete neural crest cell culture medium ${ }^{50}$. For cell growth analysis, numbers of viable cells were determined by trypan blue exclusion assay. For differentiation assay, cells were treated with $5 \mu \mathrm{M}$ all trans-retinoic acid (R2625, Sigma-Aldrich), with $0.1 \%$ DMSO as vehicle control. Phase contrast images were captured using an EVOS digital inverted microscope (Advanced Microscopy Group, Mill Creek, WA) or an Axio Observer microscope and AxioVision software (Carl Zeiss MicroImaging, Thornwood, NY).

\section{Overexpression and RNA interference}

Retroviral constructs for expressing human KDM6B (MSCV-JMJD3, Addgene \#21212) and its demethylasedefective mutant KDM6B-H1390A (MSCV-JMJD3 mutant, Addgene \#21214 $)^{51}$ were obtained from Addgene (Cambridge, MA) and verified by DNA sequencing. Lentiviral shRNA constructs targeting human KDM6B (SHC LNG-NM_001080424; shKDM6B-35, TRCN0000359975, targeting sequence, AGATTCTTTCTATGGGCTTTA; shKDM6B-36, TRCN0000367906, targeting sequence, GGAGACCTCGTGTGGATTAAT) were obtained from Sigma-Aldrich. Lentiviral constructs expressing shRNA to HOXC9 have been described previously ${ }^{38}$. BE(2)-C and SK$\mathrm{N}-\mathrm{DZ}$ cell lines with inducible expression of Myc-tagged 
HOXC9 in the absence of doxycycline (Doxy) were established as previously described ${ }^{38}$. Retroviruses were produced in 293FT cells using the packaging plasmids pHDM$\mathrm{G}$ and pMD.MLVogp, and lentiviruses were produced in 293FT cells using the packaging plasmids pLP1, pLP2, and pLP/VSVG (Thermo Fisher Scientific).

\section{Immunoblotting}

Proteins $(20-40 \mu \mathrm{g})$ were separated on SDSpolyacrylamide gels, transferred to nitrocellulose membranes, and probed with rabbit anti-HA tag (1:1000, C29F4, Cell Signaling, Danvers, MA), rabbit anti-KDM6B (1:1000, GTX124222, GeneTex, Irvine, CA), mouse antiNEFM (1:200, NF-09, sc-51683, Santa Cruz Biotechnology, Dallas, TX) or mouse anti- $\alpha$-tubulin (1:5000, B-5-1-2, Sigma-Aldrich). Histones were extracted using the EpiQuik total histone extraction kit (EpiGentek, Farmingdale, $\mathrm{NY}$ ) and analyzed by immunoblotting using mouse antihistone H3 (1:1000, 05-499, Millipore, Burlington, MA), rabbit anti-H3K27me3 (1:1000, 07-449, Millipore) or rabbit anti-H3K4me3 (1:1000, ab8895, Abcam, Cambridge, MA). Horseradish peroxidase-conjugated goat anti-mouse and anti-rabbit IgG (Santa Cruz Biotechnology) were used as secondary antibodies. Proteins were visualized using a Clarity Western ECL kit (\#1705061, Bio-RAD, Hercules, CA). For visualization using the Odyssey system (LI-COR, Lincoln, NE), goat anti-mouse IRDye 800 or 680 and anti-rabbit IRDye 800 or 680 from LI-COR were used as secondary antibodies.

\section{qRT-PCR}

Total RNA was isolated from cells using Trizol (Invitrogen, Carlsbad, CA), and reverse transcription was performed using SuperScript II Reverse Transcriptase (Invitrogen). qRT-PCR was performed using a $\mathrm{RT}^{2} \mathrm{SYBR}$ green/Fluorescein PCR master mix (Qiagen, Germantown, MD) on an iQ5 real-time PCR system (Bio-Rad) with primers against various genes (Table S3). All primer pairs were verified by melting curve analysis following qRT-PCR, with each primer pair showing a single desired amplification peak. All samples were normalized to $\beta 2$ microglobulin (B2M) mRNA levels.

\section{ChIP-qPCR}

ChIP was performed as described ${ }^{39,52}$. Cross-linked chromatin was sheared by sonication and immunoprecipitated using ChIP grade rabbit anti-HA tag (C29F4, Cell Signaling), rabbit anti-histone H3 (ab1791, Abcam), rabbit anti-H3K27me3 (07-449, Millipore), mouse anti-Myc tag (clone 4A6, Millipore) or control mouse or rabbit IgG (Santa Cruz Biotechnology). For qPCR, two independent ChIP samples were analyzed, and each sample was assayed in triplicate using primers that cover the promoter, coding and/or $3^{\prime}$ untranslated regions of $K D M 6 B$ and NEFM (Table S4). Data were presented as percentage of the input chromatin (bound/input $\times 100$ ). For ChIP against H3K27me3, data were normalized to histone H3 content obtained by anti-histone H3 ChIP.

\section{Co-immunoprecipitation (Co-IP)}

Co-IP was performed with a Dynabeads Co-IP kit (10007D, Thermo Fisher Scientific) using the detergent lysis method with extraction buffer containing $150 \mathrm{mM}$ $\mathrm{NaCl}$ and $10 \mu \mathrm{g} / \mathrm{ml}$ DNase I. BE(2)-C_tetoff-myc-HOXC9 cells were cultured in the absence of doxycycline for 6 days. Extracts from approximately $1 \times 10^{7}$ cells were incubated overnight at $4{ }^{\circ} \mathrm{C}$ with Protein G Dynabeads coated with $2 \mu \mathrm{g}$ of mouse anti-Myc tag (clone 4A6, Millipore) or mouse IgG (sc-2025, Santa Cruz Biotechnology). After washing with extraction buffer, the beads were suspended in standard SDS sample buffer and analyzed by immunoblotting using rabbit anti-KDM6B (GTX124222, GeneTex), mouse anti-Myc tag or Horseradish peroxidase-conjugated goat anti-mouse IgG (sc2005, Santa Cruz Biotechnology).

\section{Xenograft}

Approximately $5 \times 10^{6}$ neuroblastoma cells in $200 \mu \mathrm{l}$ serum-free DMEM were injected subcutaneously into flanks of 6-week-old female NOD.SCID/NCr mice (NOD.

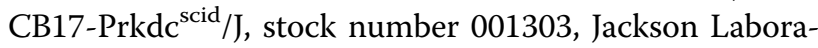
tory, Bar Harbor, ME). Approximately 4 weeks after injection, tumors were removed and weighed. All animal experiments were pre-approved by the Institutional Animal Care and Use Committee of Medical College of Georgia, Augusta University.

\section{Patient data}

Patient data used in this study were described previously $^{30,31}$. All analyses were conducted online using R2: Genomics Analysis and Visualization Platform (http://r2. amc.nl), and the resulting figures and $p$ value were downloaded.

\section{Statistics}

Quantitative data are presented as mean \pm s.d. Cell growth, qRT-PCR, and ChIP-qPCR results are representative of at least two independent experiments with each experiment being conducted in three or four technical replicates ( $n$ values in the corresponding figure legends). All quantitative data showed apparent normal distribution and equal variance. Statistical significance was estimated using Student's $t$-test (two-tailed, unpaired) for qRT-PCR and ChIP-qPCR data, and two-way ANOVA for cell growth data. Statistical analyses were conducted using Prism v7.0d for Mac (GraphPad Software, La Jolla, CA). 


\section{Acknowledgements}

We thank Rogier Versteeg and the Department of Oncogenomics at the Academic Medical Center (Amsterdam, The Netherlands) for providing the R2 Genomics Analysis and Visualization Platform. During this study, L.Y. was supported in part by the National Natural Science Foundation of China (NNSFC) grants 31672496 and 81201551, Y.Z. by the NNSFC grants 81201981 and 81550031 , H.C. by the NNSFC grant 81672502 and a grant from the National Key Research and Development Program of China (2016YFC1302204), and H.F.D. by the DoD grant W81XWH-12-1-0613 and the NIH grant R01 CA190429.

\section{Author details}

'State Key Laboratory of Silkworm Genome Biology, Southwest University, Chongqing 400716, China. ${ }^{2}$ Institute of Neural Regeneration and Repair and Department of Neurology, The First Hospital of Yichang, Three Gorges University College of Medicine, Yichang 443000, China. ${ }^{3}$ Georgia Cancer Center, Augusta University, Augusta, GA 30912, USA. ${ }^{4}$ Department of Biochemistry and Molecular, Medical College of Georgia, Augusta University, Augusta, GA 30912, USA. ${ }^{5}$ Department of Cell Biology and Anatomy, Medical College of Georgia, Augusta University, Augusta, GA 30912, USA. ${ }^{6}$ Charlie Norwood VA Medical Center, Augusta, GA 30904, USA. ${ }^{7}$ Department of Pathology, Medical College of Georgia, Augusta University, Augusta, GA 30912, USA

\section{Author contributions}

Conceptualization and design: L.Y., Y.Z., H.C., and H.-F.D. Data acquisition: L.Y., Y. Z., J.D., B.Y., M.L., and H.-F.D. Data analysis and interpretation: L.Y., Y.Z., H.C., C.Y., Z.D., and H.F.D. Manuscript writing: H.-F.D., with contributions from L.Y., Y.Z., and H.C.

\section{Conflict of interest}

The authors declare that they have no conflict of interest.

\section{Publisher's note}

Springer Nature remains neutral with regard to jurisdictional claims in published maps and institutional affiliations.

Supplementary Information accompanies this paper at (https://doi.org/ 10.1038/s41389-018-0112-0).

Received: 20 September 2018 Revised: 19 November 2018 Accepted: 11 December 2018

Published online: 04 January 2019

\section{References}

1. Brodeur, G. M. Neuroblastoma: biological insights into a clinical enigma. Nat. Rev. Cancer 3, 203-216 (2003).

2. Maris, J. M., Hogarty, M. D., Bagatell, R. \& Cohn, S. L. Neuroblastoma. Lancet 369, 2106-2120 (2007).

3. Cheung, N. K. \& Dyer, M. A. Neuroblastoma: developmental biology, cancer genomics and immunotherapy. Nat. Rev. Cancer 13, 397-411 (2013).

4. Marshall, G. M. et al. The prenatal origins of cancer. Nat. Rev. Cancer 14, 277-289 (2014)

5. Cohn, S. L. et al. The International Neuroblastoma Risk Group (INRG) classification system: an INRG Task Force report. J. Clin. Oncol. 27, 289-297 (2009).

6. Park, J. R. et al. Children's Oncology Group's 2013 blueprint for research: neuroblastoma. Pediatr. Blood. Cancer 60, 985-993 (2013).

7. Pinto, N. R. et al. Advances in risk classification and treatment strategies for neuroblastoma. J. Clin. Oncol. 33, 3008-3017 (2015).

8. Shimada, H. et al. The international neuroblastoma pathology classification (the Shimada system). Cancer 86, 364-372 (1999).

9. Sidell, N. Retinoic acid-induced growth inhibition and morphologic differentiation of human neuroblastoma cells in vitro. J. Natl Cancer Inst. 68 589-596 (1982).

10. Sidell, N., Altman, A., Haussler, M. R. \& Seeger, R. C. Effects of retinoic acid (RA) on the growth and phenotypic expression of several human neuroblastoma cell lines. Exp. Cell Res. 148, 21-30 (1983).
11. Reynolds, C. P., Matthay, K. K., Villablanca, J. G. \& Maurer, B. J. Retinoid therapy of high-risk neuroblastoma. Cancer Lett. 197, 185-192 (2003).

12. Volchenboum, S. L. \& Cohn, S. L. Progress in defining and treating high-risk neuroblastoma: lessons from the bench and bedside. J. Clin. Oncol. 27, 1003-1004 (2009).

13. Matthay, K. K. et al. Treatment of high-risk neuroblastoma with intensive chemotherapy, radiotherapy, autologous bone marrow transplantation, and 13-cis-retinoic acid. Children's Cancer Group. N. Engl. J. Med. 341, 1165-1173 (1999).

14. Matthay, K. K. et al. Long-term results for children with high-risk neuroblastoma treated on a randomized trial of myeloablative therapy followed by 13-cis-retinoic acid: a children's oncology group study. J. Clin. Oncol. 27, 1007-1013 (2009).

15. Laugesen, A. \& Helin, K. Chromatin repressive complexes in stem cells, development, and cancer. Cell Stem. Cell 14, 735-751 (2014).

16. Surface, L. E., Thornton, S. R. \& Boyer, L. A. Polycomb group proteins set the stage for early lineage commitment. Cell Stem. Cell 7, 288-298 (2010).

17. Hirabayashi, Y. \& Gotoh, Y. Epigenetic control of neural precursor cell fate during development. Nat. Rev. Neurosci. 11, 377 (2010).

18. Margueron, R. \& Reinberg, D. The Polycomb complex PRC2 and its mark in life. Nature 469, 343-349 (2011).

19. Wang, C. et al. EZH2 mediates epigenetic silencing of neuroblastoma suppressor genes CASZ1, CLU, RUNX3, and NGFR. Cancer Res. 72, 315-324 (2012).

20. Tsubota, S. et al. PRC2-mediated transcriptomic alterations at the embryonic stage govern tumorigenesis and clinical outcome in MYCN-driven neuroblastoma. Cancer Res. 77, 5259-5271 (2017).

21. Li, Z. et al. EZH2 regulates neuroblastoma cell differentiation via NTRK1 promoter epigenetic modifications. Oncogene 37, 2714-2727 (2018).

22. Black, J. C., Van Rechem, C. \& Whetstine, J. R. Histone lysine methylation dynamics: establishment, regulation, and biological impact. Mol. Cell 48, 491-507 (2012)

23. Mosammaparast, N. \& Shi, Y. Reversal of histone methylation: biochemical and molecular mechanisms of histone demethylases. Annu. Rev. Biochem. 79, 155-179 (2010).

24. Swigut, T. \& Wysocka, J. H3K27 demethylases, at long last. Cell 131, 29-32 (2007).

25. Liu, M. et al. Transcriptional profiling reveals a common metabolic program in high-risk human neuroblastoma and mouse neuroblastoma sphere-forming cells. Cell Rep. 17, 609-623 (2016).

26. Weiss, W. A., Aldape, K., Mohapatra, G., Feuerstein, B. G. \& Bishop, J. M. Targeted expression of MYCN causes neuroblastoma in transgenic mice. EMBO J. 16, 2985-2995 (1997).

27. Moore, H. C. et al. Histological profile of tumours from MYCN transgenic mice J. Clin. Pathol. 61, 1098-1103 (2008).

28. Teitz, T. et al. Preclinical models for neuroblastoma: establishing a baseline for treatment. PLoS One 6, e19133 (2011).

29. Hansford, L. M. et al. Mechanisms of embryonal tumor initiation: distinct roles for MycN expression and MYCN amplification. Proc. Natl Acad. Sci. USA 101, 12664-12669 (2004)

30. Zhang, W. et al. Comparison of RNA-seq and microarray-based models for clinical endpoint prediction. Genome Biol. 16, 133 (2015).

31. Kocak, H. et al. Hox-C9 activates the intrinsic pathway of apoptosis and is associated with spontaneous regression in neuroblastoma. Cell Death Dis. $\mathbf{4}$, e586 (2013).

32. da Silva, J. S. \& Dotti, C. G. Breaking the neuronal sphere: regulation of the actin cytoskeleton in neuritogenesis. Nat. Rev. Neurosci. 3, 694-704 (2002).

33. Luo, L. Actin cytoskeleton regulation in neuronal morphogenesis and structural plasticity. Annu. Rev. Cell Dev. Biol. 18, 601-635 (2002).

34. Santy, L. C. \& Casanova, J. E. GTPase signaling: bridging the GAP between ARF and Rho. Curr. Biol. 12, R360-R362 (2002).

35. Jackson, T. R., Kearns, B. G. \& Theibert, A. B. Cytohesins and centaurins: mediators of PI 3-kinase-regulated Arf signaling. Trends Biochem. Sci. 25, 489-495 (2000).

36. Hahn, C. K. et al. Expression-based screening identifies the combination of histone deacetylase inhibitors and retinoids for neuroblastoma differentiation. Proc. Natl Acad. Sci. USA 105, 9751-9756 (2008).

37. Huang, S. et al. ZNF423 is critically required for retinoic acid-induced differentiation and is a marker of neuroblastoma outcome. Cancer Cell 15, 328-340 (2009).

38. Mao, L. et al. HOXC9 links cell-cycle exit and neuronal differentiation and is a prognostic marker in neuroblastoma. Cancer Res. 71, 4314-4324 (2011). 
39. Wang, X. et al. HOXC9 directly regulates distinct sets of genes to coordinate diverse cellular processes during neuronal differentiation. BMC Genom. 14, 830 (2013).

40. Zha, Y. et al. Functional dissection of HOXD cluster genes in regulation of neuroblastoma cell proliferation and differentiation. PLoS One 7, e40728 (2012).

41. Laser-Azogui, A., Kornreich, M., Malka-Gibor, E. \& Beck, R. Neurofilament assembly and function during neuronal development. Curr. Opin. Cell Biol. 32, 92-101 (2015).

42. Ernsberger, U. The role of GDNF family ligand signalling in the differentiation of sympathetic and dorsal root ganglion neurons. Cell Tissue Res. 333, 353-371 (2008).

43. Bosselut, R. Pleiotropic functions of H3K27Me3 demethylases in immune cell differentiation. Trends Immunol. 37, 102-113 (2016).

44. Pearson, J. C., Lemons, D. \& McGinnis, W. Modulating Hox gene functions during animal body patterning. Nat. Rev. Genet. 6, 893-904 (2005).

45. Svingen, T. \& Tonissen, K. F. Hox transcription factors and their elusive mammalian gene targets. Heredity 97, 88-96 (2006).
46. Lee, S., Lee Jae, W. \& Lee, S.-K. UTX, a histone H3-lysine 27 demethylase, acts as a critical switch to activate the cardiac developmental program. Dev. Cell 22 , 25-37 (2012).

47. Burgold, T. et al. The histone H3 lysine 27-specific demethylase Jmjd3 is required for neural commitment. PLoS One 3, e3034 (2008).

48. Park, D. H. et al. Activation of neuronal gene expression by the JMJD3 demethylase is required for postnatal and adult brain neurogenesis. Cell Rep. $\mathbf{8}$, 1290-1299 (2014).

49. Ntziachristos, P. et al. Contrasting roles of histone 3 lysine 27 demethylases in acute lymphoblastic leukaemia. Nature 514, 513-517 (2014).

50. Etchevers, H. Primary culture of chick, mouse or human neural crest cells. Nat. Protoc. 6, 1568-1577 (2011).

51. Sen, G. L., Webster, D. E., Barragan, D. I., Chang, H. Y. \& Khavari, P. A. Control of differentiation in a self-renewing mammalian tissue by the histone demethylase JMJD3. Genes Dev. 22, 1865-1870 (2008).

52. Ding, J. et al. The histone $H 3$ methyltransferase G9A epigenetically activates the serine-glycine synthesis pathway to sustain cancer cell survival and proliferation. Cell Metab. 18, 896-907 (2013). 